\title{
Álgebra linear numérica: aplicações em métodos computacionais e sua importância para a engenharia
}

\author{
José Rhuan de Souza Pereira, Renato Brito Costa
}

\begin{abstract}
Resumo - O presente trabalho tem como objetivo validar a importância da álgebra linear em métodos computacionais e cursos de engenharia, mostrando não só aspectos teóricos, como também aplicações práticas da disciplina. Apesar dos conceitos desta área matemática serem amplamente difundidos em seu campo teórico, muitas vezes sua aplicabilidade passa despercebida, principalmente em semestres iniciais dos cursos de engenharia, contribuindo assim, para uma difícil visualização de sua usabilidade. Desta forma, surge a necessidade de demonstrar como os conceitos se aplicam na resolução de problemas enfrentados pelos engenheiros. De fato, estas aplicações podem ser vistas não só na computação, como também em outras engenharias. Sendo assim, é fundamental a abordagem de alguns destes usos, bem como seus respectivos métodos de execução.
\end{abstract}

Palavras-chave - Álgebra; Engenharia; Computação; Aplicação.

\section{Introdução}

A álgebra linear é uma vertente da matemática de extrema importância para os cursos de engenharia. Os modelos lineares assumiram bastante importância no estudo da computação e informática a medida que estas áreas passaram a evoluir e se tornaram cada vez mais complexas. Nos cursos de engenharia, esta disciplina é encontrada em semestres iniciais, pois é um importante constituinte para aquisição de uma base sólida que possibilitará ao estudante diversas ferramentas para resolução de problemas e uma melhor análise de modelos e sistemas. Aplicações práticas da disciplina podem ser notadas em algumas situações como: computação gráfica, criptografia, sistemas de controle, reconhecimento facial, edição de imagens, programação linear, cálculo estocástico, dentre outras.

\section{Aplicações}

\subsection{Computação Gráfica}

A computação gráfica é a área da computação que tem como objetivo principal a análise, processamento e síntese de imagens, sendo de fundamental importância no desenvolvimento de animações, modelos tridimensionais, entre diversos outros usos. Segundo a ISO (International Organization for Standardization), temos como definição: "um conjunto de ferramentas e técnicas para conversão de dados de/para dispositivos gráficos." Representação vetorial: esta representação é empregada principalmente em computação gráfica em que são considerados como elementos básicos constituintes de uma imagem os pontos, linhas e curvas, ou seja características que irão descrever a forma do objeto. Como o computador trabalha com números binários, este interpreta as imagens como sendo uma descrição matricial constituída por estes números. Sendo assim, os pixels são representados por matrizes formadas apenas pelos números 0 ou 1.Para alterarmos alguma imagem, são utilizadas as chamadas transformações geométricas. Desta forma podemos manipular características, como cor, tamanho e disposição no plano. Cada ponto em um sistema de coordenadas pode ser associado a uma matriz que lhe represente. Algumas transformações importantes devem ser abordadas mais a fundo, tais como: Translação: este é o nome dado ao ato de mudar um objeto de posição. Para efetuar a translação de 
pontos no plano $(\mathrm{x}, \mathrm{y})$, devemos adicionar um vetor de deslocamento à sua posição atua. Desta forma teremos uma nova posição dada pela exemplificação seguinte:

$\mathrm{X}^{\prime}=\mathrm{x}+\mathrm{Tx}$

$\mathrm{Y}^{\prime}=\mathrm{y}+\mathrm{Ty}$

Mudança de escala: quando alteramos uma escala, a imagem é definida por um novo conjunto de ponto de diferente tamanho. Para fazermos isto, devemos multiplicar suas coordenadas por um escalar, da seguinte forma:

$\mathrm{X}^{\prime}=\mathrm{x} * \mathrm{Ax}$

$\mathrm{Y}^{\prime}=\mathrm{y}^{*} \mathrm{Ay}$

Onde, $x$ ' e y' representam as novas coordenadas da imagem com a mudança de escala e "A" representa a grandeza escalar.

$\mathrm{Na}$ forma matricial, esta transformação pode ser representada da seguinte forma:

$\left[\begin{array}{ll}x & y\end{array}\right]\left[\begin{array}{cc}A x & 0 \\ 0 & A y\end{array}\right]$

Rotação: em outras palavras significa girar. Se rotacionarmos um ponto em torno de um ângulo $\theta$ em torno de sua origem teremos como novos pontos os resultados das expressões abaixo:

$\mathrm{X}^{\prime}=\mathrm{x} \cos (\theta)-\mathrm{y} \operatorname{sen}(\theta)$

$Y^{\prime}=y \cos (\theta)-x \operatorname{sen}(\theta)$

Rotação em torno do eixo coordenado $\mathrm{X}$ em $\theta$ unidades angulares;

A transformação linear $\mathrm{T}: \mathrm{R}^{3} \rightarrow \mathrm{R}^{3}$, dada por $T_{\theta, x}(\mathrm{x}, \mathrm{y}, \mathrm{z})=(\mathrm{x}, \mathrm{y} \cos \theta-\mathrm{z} \operatorname{sen} \theta, \quad \mathrm{y} \operatorname{sen} \theta+\mathrm{z} \cos \theta)$ é denominada de rotação em torno do eixo coordenado $\mathrm{x}$. $\mathrm{Na}$ forma matricial, temos que:

$$
T\left[\begin{array}{l}
x \\
y \\
z
\end{array}\right]=\left[\begin{array}{ccc}
1 & 0 & 0 \\
0 & \cos \theta & -\operatorname{sen} \theta \\
0 & \operatorname{sen} \theta & \cos \theta
\end{array}\right]\left[\begin{array}{l}
x \\
y \\
z
\end{array}\right]=\left[\begin{array}{c}
x \\
y \cos \theta-z \operatorname{sen} \theta \\
y \operatorname{sen} \theta+z \cos \theta
\end{array}\right]
$$

A matriz que representa a rotação $T_{\ominus, x}$ é dada por:

$$
\left[\begin{array}{ccc}
1 & 0 & 0 \\
0 & \cos \theta & -\operatorname{sen} \theta \\
0 & \operatorname{sen} \theta & \cos \theta
\end{array}\right]
$$

Rotação em torno do eixo coordenado $\mathrm{Y}$ em $\theta$ unidades angulares;

A transformação linear $\mathrm{T}: \mathrm{R}^{3} \rightarrow \mathrm{R}^{3}$, dada por $\mathrm{T}_{\boldsymbol{\theta}, \mathrm{y}}(\mathrm{x}, \mathrm{y}, \mathrm{z})=(\mathrm{x} \cos \theta+\mathrm{z} \operatorname{sen} \theta, \mathrm{y},-\mathrm{x} \operatorname{sen} \theta+\mathrm{z} \cos \theta)$ é denominada de rotação em torno do eixo coordenado y. Na forma matricial, temos que:

$$
T\left[\begin{array}{l}
x \\
y \\
z
\end{array}\right]=\left[\begin{array}{ccc}
\cos \theta & 0 & \operatorname{sen} \theta \\
0 & 1 & 0 \\
-\operatorname{sen} \theta & 0 & \cos \theta
\end{array}\right]\left[\begin{array}{l}
x \\
y \\
z
\end{array}\right]=\left[\begin{array}{c}
x \cos \theta+z \operatorname{sen} \theta \\
y \\
-x \operatorname{sen} \theta+z \cos \theta
\end{array}\right]
$$

A matriz que representa a rotação $T_{\theta, y}$ é dada por:

$$
\left[\begin{array}{ccc}
\cos \theta & 0 & \operatorname{sen} \theta \\
0 & 1 & 0 \\
-\operatorname{sen} \theta & 0 & \cos \theta
\end{array}\right]
$$

Rotação em torno do eixo coordenado $\mathrm{Z}$ em $\theta$ unidades angulares;

A transformação linear $\mathrm{T}: \mathrm{R}^{3} \rightarrow \mathrm{R}^{3}$, dada por $T_{\theta_{z} z}(\mathrm{x}, \mathrm{y}, \mathrm{z})=(\mathrm{x} \cos \theta-\mathrm{y} \operatorname{sen} \theta, \mathrm{x} \operatorname{sen} \theta+\mathrm{y} \cos \theta, \mathrm{z})$ é denominada de rotação em 24 torno do eixo coordenado z. Na forma matricial, temos que:

$$
T\left[\begin{array}{l}
x \\
y \\
z
\end{array}\right]=\left[\begin{array}{ccc}
\cos \theta & -\operatorname{sen} \theta & 0 \\
\operatorname{sen} \theta & \cos \theta & 0 \\
0 & 0 & 1
\end{array}\right]\left[\begin{array}{l}
x \\
y \\
z
\end{array}\right]=\left[\begin{array}{c}
x \cos \theta-y \operatorname{sen} \theta \\
x \operatorname{sen} \theta+y \cos \theta \\
z
\end{array}\right]
$$


A matriz que representa a rotação $T_{\ominus, z}$ é dada por:

$$
\left[\begin{array}{ccc}
\cos \theta & -\operatorname{sen} \theta & 0 \\
\operatorname{sen} \theta & \cos \theta & 0 \\
0 & 0 & 1
\end{array}\right]
$$

Reflexão em torno do eixo $\mathrm{x}$ :

A transformação linear $\mathrm{T}: \mathrm{R}^{2} \rightarrow \mathrm{R}^{2}$, dada por $\mathrm{T}(\mathrm{x}, \mathrm{y})=(\mathrm{x},-\mathrm{y})$ é denominada de reflexão em torno do eixo x. Na forma matricial, temos que:

$$
T\left[\begin{array}{l}
x \\
y
\end{array}\right]=\left[\begin{array}{c}
x \\
-y
\end{array}\right]=\left[\begin{array}{cc}
1 & 0 \\
0 & -1
\end{array}\right]\left[\begin{array}{l}
x \\
y
\end{array}\right]
$$

Neste caso, diz-se que a matriz $\left[\begin{array}{cc}1 & 0 \\ 0 & -1\end{array}\right]$ representa a transformação T.

Reflexão em torno do eixo y:

A transformação linear $\mathrm{T}: \mathrm{R}^{2} \rightarrow \mathrm{R}^{2}$ dada por $\mathrm{T}(\mathrm{x}, \mathrm{y})=(-\mathrm{x}, \mathrm{y})$ é denominada de reflexão em torno do eixo y. Na forma matricial temos que:

$T\left[\begin{array}{l}x \\ y\end{array}\right]=\left[\begin{array}{c}-x \\ y\end{array}\right]=\left[\begin{array}{cc}-1 & 0 \\ 0 & 1\end{array}\right]\left[\begin{array}{l}x \\ y\end{array}\right]$

Neste caso, diz-se que a matriz $\left[\begin{array}{cc}-1 & 0 \\ 0 & 1\end{array}\right]$ representa a transformação T.

2.2 Reflexão em torno da reta $\mathrm{y}=\mathrm{x}$ :

A transformação linear $\mathrm{T}: \mathrm{R}^{2} \rightarrow \mathrm{R}^{2}$ dada por $\mathrm{T}(\mathrm{x}, \mathrm{y})=(\mathrm{y}, \mathrm{x})$ é denominada de reflexão em torno da reta $y=x$. Na forma matricial, temos que:

$T\left[\begin{array}{l}x \\ y\end{array}\right]=\left[\begin{array}{l}y \\ x\end{array}\right]=\left[\begin{array}{ll}0 & 1 \\ 1 & 0\end{array}\right]\left[\begin{array}{l}x \\ y\end{array}\right]$

Neste caso, diz-se que a matriz $\left[\begin{array}{ll}0 & 1 \\ 1 & 0\end{array}\right]$ representa a transformação T.

Reflexão em torno da reta $\mathrm{y}=-\mathrm{x}$ :

A transformação linear $\mathrm{T}: \mathrm{R}^{2} \rightarrow \mathrm{R}^{2}$ dada por $\mathrm{T}(\mathrm{x}, \mathrm{y})=(-\mathrm{y},-\mathrm{x})$ é denominada de reflexão em torna da reta $y=-x$. Na forma matricial, temos que:

$T\left[\begin{array}{l}x \\ y\end{array}\right]=\left[\begin{array}{l}-y \\ -x\end{array}\right]=\left[\begin{array}{cc}0 & -1 \\ -1 & 0\end{array}\right]\left[\begin{array}{l}x \\ y\end{array}\right]$

Neste caso, diz-se que a matriz $\left[\begin{array}{cc}0 & -1 \\ -1 & 0\end{array}\right]$ representa a transformação T.

Reflexão com relação a origem;

A transformação linear $\mathrm{T}: \mathrm{R}^{2} \rightarrow \mathrm{R}^{2}$ dada por $\mathrm{T}(\mathrm{x}, \mathrm{y})=(-\mathrm{x},-\mathrm{y})$ é denominada de reflexão com relação a origem. Na forma matricial, temos que : 
$T\left[\begin{array}{l}x \\ y\end{array}\right]=\left[\begin{array}{l}-x \\ -y\end{array}\right]=\left[\begin{array}{cc}-1 & 0 \\ 0 & -1\end{array}\right]\left[\begin{array}{l}x \\ y\end{array}\right]$

Neste caso, diz-se que a matriz $\left[\begin{array}{cc}-1 & 0 \\ 0 & -1\end{array}\right]$ representa a transformação T.

Dilatação ou contração;

A transformação linear $\mathrm{T}: \mathrm{R}^{2} \rightarrow \mathrm{R}^{2}$ dada por $\mathrm{T}(\mathrm{x}, \mathrm{y})=\mathrm{k}(\mathrm{x}, \mathrm{y})$ é denominada de dilatação ou contração. Na forma matricial, temos que:

$$
T\left[\begin{array}{l}
x \\
y
\end{array}\right]=\left[\begin{array}{l}
k x \\
k y
\end{array}\right]=\left[\begin{array}{ll}
k & 0 \\
0 & k
\end{array}\right]\left[\begin{array}{l}
x \\
y
\end{array}\right]
$$

Se $|k|>1$, T é uma dilatação;

Se $|k|<1$, T é uma contração;

Se $\mathrm{k}=1$, $\mathrm{T}$ é a identidade;

Se $\mathrm{k}<0$, $\mathrm{T}$ troca o sentido do vetor.

Neste caso, diz-se que a matriz $\left[\begin{array}{ll}k & 0 \\ 0 & k\end{array}\right]$ representa a transformação T.

Cisalhamento na direção do eixo $\mathrm{x}$;

A transformação linear $\mathrm{T}: \mathrm{R}^{2} \rightarrow \mathrm{R}^{2}$ dada por $\mathrm{T}(\mathrm{x}, \mathrm{y})=(\mathrm{x}+\mathrm{ky}, \mathrm{y})$ é denominada de cisalhamento na direção do eixo x. Na forma matricial, temos que:

$$
T\left[\begin{array}{l}
x \\
y
\end{array}\right]=\left[\begin{array}{c}
x+k y \\
y
\end{array}\right]=\left[\begin{array}{ll}
1 & k \\
0 & 1
\end{array}\right]\left[\begin{array}{l}
x \\
y
\end{array}\right]
$$

Neste caso, diz-se que a matriz $\left[\begin{array}{ll}1 & k \\ 0 & 1\end{array}\right]$ representa a transformação T.

Cisalhamento na direção do eixo y;

A transformação $\mathrm{T}: \mathrm{R}^{2} \rightarrow \mathrm{R}^{2}$ dada por $\mathrm{T}(\mathrm{x}, \mathrm{y})=(\mathrm{x}, \mathrm{y}+\mathrm{kx})$ é denominada de cisalhamento na direção do eixo y. Na forma matricial, temos que:

$$
T\left[\begin{array}{l}
x \\
y
\end{array}\right]=\left[\begin{array}{c}
x \\
y+k x
\end{array}\right]=\left[\begin{array}{ll}
1 & 0 \\
k & 1
\end{array}\right]\left[\begin{array}{l}
x \\
y
\end{array}\right]
$$

Neste caso, diz-se que a matriz $\left[\begin{array}{ll}1 & 0 \\ k & 1\end{array}\right]$ representa a transformação T.

\subsection{Criptografia}

A criptografia consiste no estudo de técnicas e princípios capazes de transformar uma determinada informação em algo ilegível, de forma que esta possa ser reconhecida apenas pelo seu destinatário.

Cifras de Substituição:

É um método de criptografia que consiste em um sistema pré-definido de substituição. Para criptografar uma mensagem, as letras de uma frase são substituídas de modo a formar uma cifra. Temos a Cifra de César como um exemplo deste método. A Cifra de César é um dos mais 
simples e conhecidos métodos de criptografia, que consiste na substituição de uma letra por outra trocando apenas sua posição. Neste método são utilizadas chaves de ciframento, que são números que definem a quantidade de deslocamento da mensagem original em relação à mensagem cifrada. Por exemplo a mensagem: "Devemos atacar logo", cifra com a chave ROT4 ficará assim:" Hiziqsw exegev psks", ou seja cada letra do alfabeto original é trocada pela letra seguinte em 4 posições.

Figura 1 - Ilustração da correspondência entre letras

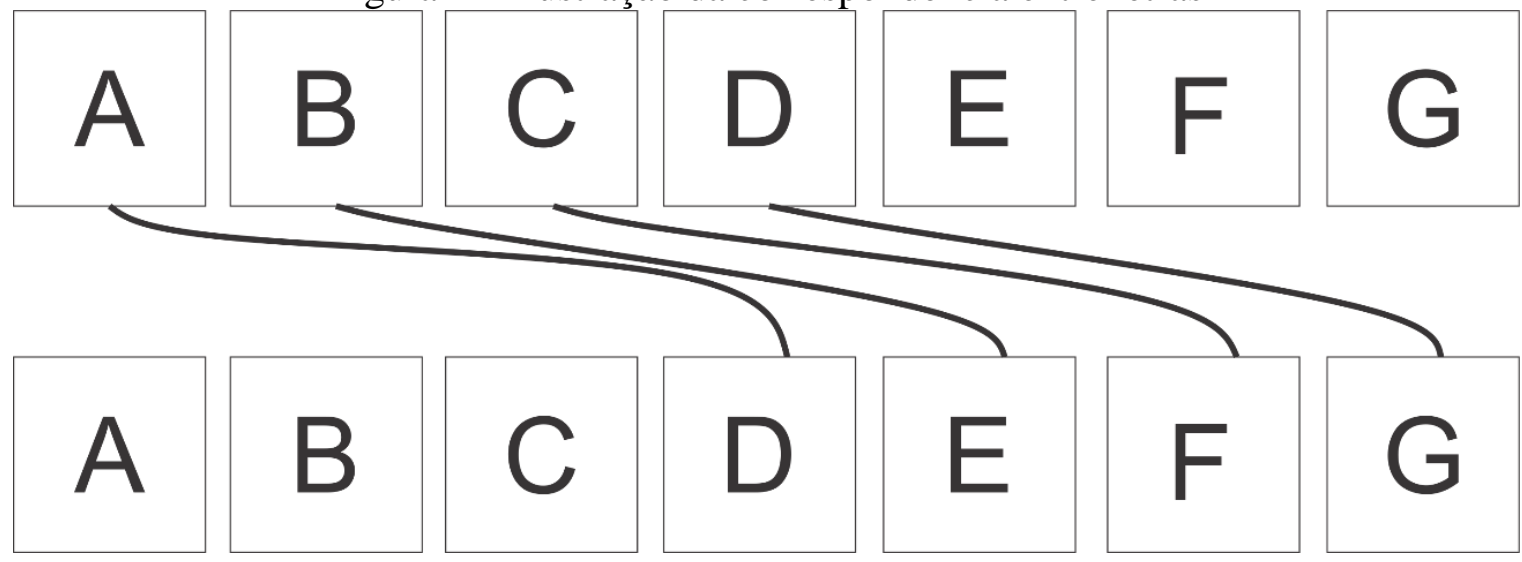

Criptografia por Matrizes:

O método de criptografia por matrizes funciona da seguinte forma: a mensagem a ser criptografada é convertida em uma matriz na qual cada letra é transformada em um número conforme sua posição no alfabeto. As posições em que não houverem números são preenchidas por 0 .

Em álgebra linear, uma matriz pode ser utilizada para criptografar uma mensagem de maneira simples. Seja A a matriz contendo a mensagem a ser criptografada e B a matriz com a chave de criptografia, basta transformarmos as duas matrizes, em matrizes numéricas e depois multiplicar seus elementos, gerando assim uma nova matriz contendo a mensagem criptografada. Utilizaremos como exemplo a frase :"Vamos pela manhã" e como chave de criptografia a palavra "Estrategistas".

$\left[\begin{array}{cccc}v & a & m & o \\ s & p & e & l \\ a & m & a & n \\ h & a & - & -\end{array}\right]\left[\begin{array}{llll}e & s & t & r \\ a & t & e & - \\ g & i & s & t \\ a & s & - & -\end{array}\right] \rightarrow\left[\begin{array}{cccc}22 & 1 & 13 & 15 \\ 19 & 16 & 5 & 12 \\ 1 & 13 & 1 & 14 \\ 8 & 1 & 0 & 0\end{array}\right]\left[\begin{array}{cccc}5 & 19 & 20 & 18 \\ 1 & 20 & 5 & 0 \\ 7 & 9 & 19 & 20 \\ 1 & 19 & 0 & 0\end{array}\right]$

$=\left[\begin{array}{cccc}217 & 840 & 692 & 656 \\ 158 & 954 & 555 & 442 \\ 39 & 554 & 104 & 38 \\ 41 & 172 & 165 & 144\end{array}\right]$

Para decodificar a mensagem, basta multiplicar a matriz codificada pela inversa da matriz que representa a chave de encriptação, assim: 


$$
\left[\begin{array}{cccc}
217 & 840 & 692 & 656 \\
158 & 954 & 555 & 442 \\
39 & 554 & 104 & 38 \\
41 & 172 & 165 & 144
\end{array}\right] \times\left[\begin{array}{cccc}
\frac{-950}{1751} & \frac{551}{1751} & \frac{855}{1751} & \frac{-35}{1751} \\
\frac{50}{1751} & \frac{-29}{1751} & \frac{-45}{1751} & \frac{94}{1751} \\
\frac{-10}{1751} & \frac{356}{1751} & \frac{9}{1751} & \frac{-369}{1751} \\
\frac{639}{1751} & \frac{-518}{1751} & \frac{-200}{1751} & \frac{641}{1751}
\end{array}\right]=\left[\begin{array}{cccc}
22 & 1 & 13 & 15 \\
19 & 16 & 5 & 12 \\
1 & 13 & 1 & 14 \\
8 & 1 & 0 & 0
\end{array}\right]
$$

A partir disto, é necessário somente converter os números para letras de acordo com suas respectivas posições no alfabeto.

\subsection{Circuitos Elétricos}

Os circuitos elétricos são importantes componentes de equipamentos eletrônicos tais como processadores, memórias, dentre outros. Um circuito é chamado simples, quando é formado por apenas uma corrente, sendo assim é necessária somente uma equação para descrevê-lo. Já quando é formado por $\mathrm{n}$ correntes serão necessárias $\mathrm{n}$ equações lineares e assim o problema se torna mais complexo. Para que se tenha uma solução do sistema é realizado o escalonamento e assim obteremos os valores das incógnitas. Para abordarmos este assunto com mais profundidade faz-se necessária a elucidação de algumas leis, sendo elas a lei de Ohm e a lei de Kirchhoff.. A lei de Ohm nos dá a seguinte equação: $\mathrm{E}=\mathrm{RI}$, em que $\mathrm{E}$ representa a força elétrica, $\mathrm{R}$ representa a resistência $\mathrm{e}$ I representa a corrente. Já em relação as leis de Kirchhoff. Temos duas, a lei da corrente e a lei da voltagem. A primeira estabelece que a soma das correntes que entram em qualquer nó é igual à soma das correntes que saem dele. Já a segunda estabelece que a soma das quedas de voltagem ao longo de qualquer circuito é igual à voltagem do circuito (fornecido por uma bateria). Temos o circuito abaixo como exemplo, no qual deseja-se determinar as correntes:

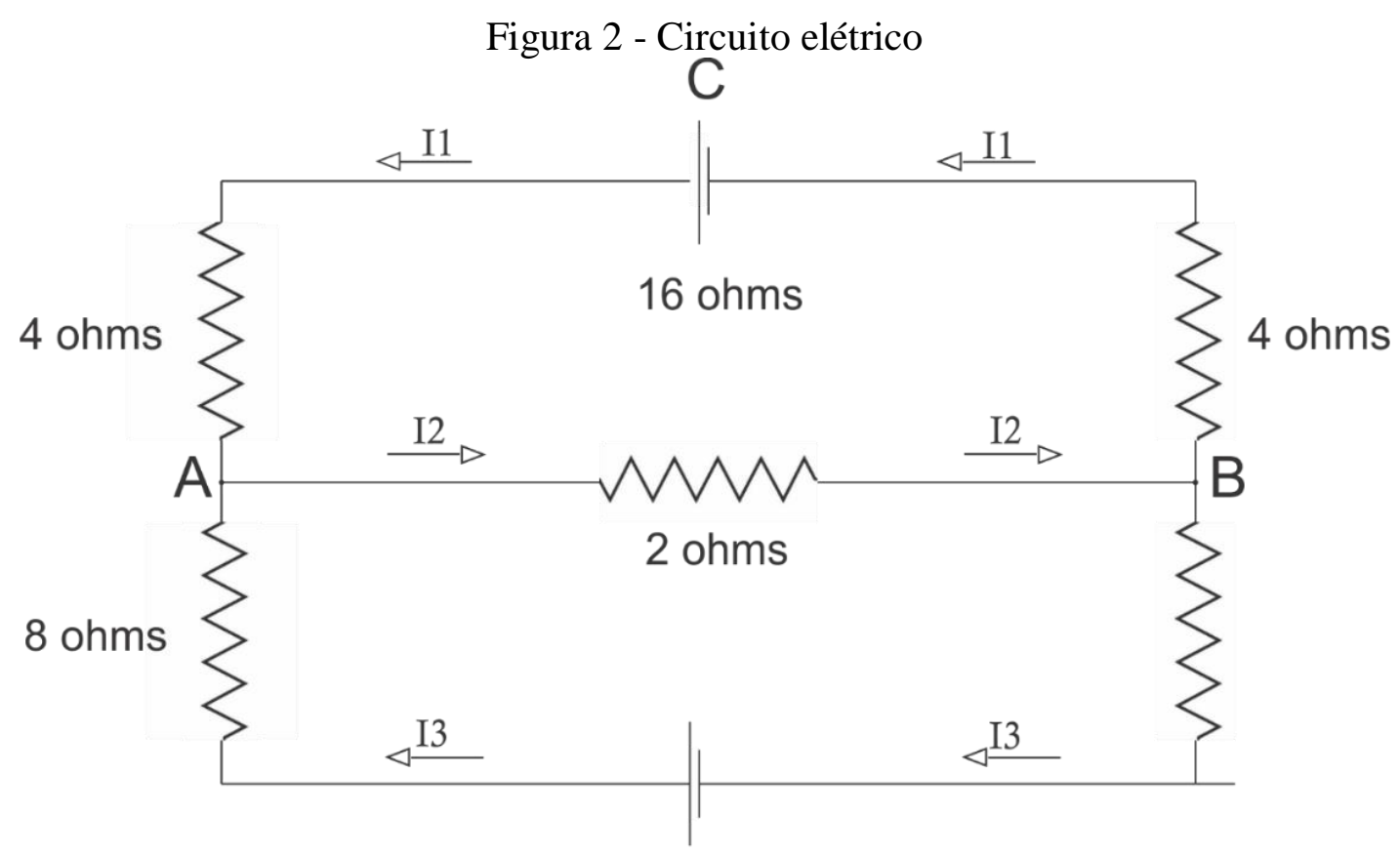

32 ohms

Nó A: $\mathrm{I} 1-\mathrm{I} 2+\mathrm{I} 3=0$

Nó B: $\mathrm{I} 1-\mathrm{I} 2+\mathrm{I} 3=0$

Circuito CABC: $811+12=16$ 
Circuito DABD: $12+413=32$

Assim, temos o sistema linear formado, representado na forma de matriz:

$\left(\begin{array}{cccc}1 & -1 & 1 & 0 \\ 8 & 2 & 0 & 16 \\ 0 & 2 & 8 & 32\end{array}\right)$

Resolvendo este sistema, encontramos os seguintes valores para as correntes:

$\mathrm{I} 1=1$

$\mathrm{I} 2=4$

$\mathrm{I} 2=3$

\subsection{Inteligência Artificial}

A inteligência artificial é uma área que se encontra em grande ascensão. São notáveis os esforços para aperfeiçoamento deste tipo de tecnologia. Grandes empresas já utilizam-na para diversos usos como observar comportamentos relacionados a consumo de produtos e serviços a partir do processamento de uma grande quantidade de dados. Um problema muito importante para a aprendizagem de máquina é a classificação, que é a base central da aprendizagem de máquina.

A classificação é uma das funções que podem ser atribuídas às redes neurais. Para uma melhor compreensão, uma introdução sobres este tópico será muito elucidativa. Redes Neurais são modelos computacionais construídos tendo como inspiração o sistema nervoso animal. Estas têm uma importante habilidade de se adaptar ao ambiente, melhorando assim seu desempenho, sendo responsável pela aprendizagem de máquina ao receber uma entrada, realizar um processamento e retornar uma saída, tendo diversas utilidades como biometria, diagnóstico médico e reconhecimento de voz. Retornando ao problema da classificação sua tarefa é selecionar uma entrada e dar a ela um rótulo para sua identificação. Há um grande conjunto de exemplos chamados de conjuntos de treinamento que já foram classificados para que o algoritmo saiba reconhecer futuras variações. Dessa forma, a máquina deverá ser capaz de identificar novas entradas por si própria. Podemos citar como exemplo da aplicação da álgebra linear um classificador logístico, que usaremos para "ensinar " à máquina como reconhecer uma letra em diferentes situações. Funciona da seguinte forma: É feita uma entrada, como por exemplo, os pixels de uma imagem e aplicada uma função linear a eles para gerar as previsões.

Uma função linear é apenas uma multiplicação de matrizes que considera todas as entradas como um grande vetor X e multiplica por uma matriz para gerar previsões, uma para cada classe de saída. Chamaremos a entrada de X, os pesos de W, e o termo de tendência de b. Os pesos da matriz e a tendência são onde entra a aprendizagem de máquina. Tentaremos encontrar valores para os pesos e tendências que são bons em fazer previsões.

Figura 3 - Ilustração para pesos e tendências necessários para se fazer previsões

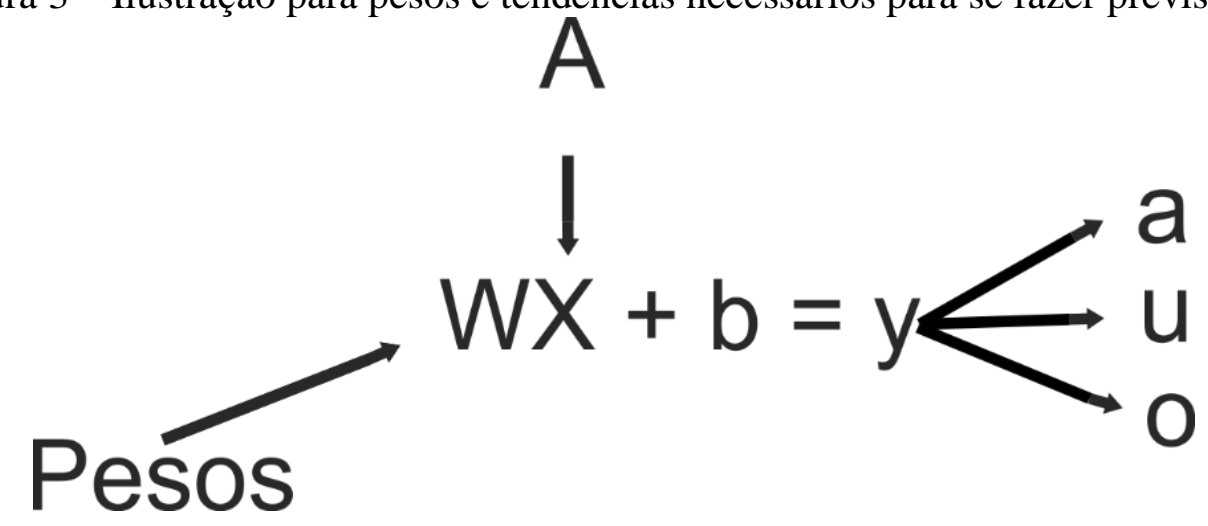


Mas como utilizar as notas para realizar a classificação? Cada imagem de entrada pode ter apenas um rótulo, então será necessário transformar as notas em probabilidades. É necessário que a probabilidade da classe correta seja próxima de 1 e a probabilidade das outras classes seja próxima de 0. A forma de transformar notas em probabilidades é usar uma função softmax que denotaremos por S. Vale dissertar um pouco sobre esta função: uma função softmax é utilizada em redes neurais com o objetivo de classificação, com ela uma rede neural apresenta a probabilidade do dado ser de uma determinada classe definida. Também há outros tipos de funções tais como: função linear, função com limite (Threshold), Sigmoid, que são chamadas de funções de ativação, porém este tópico não será abordado aqui com mais aprofundamento pois foge ao objetivo do presente trabalho. É importante saber que esta fórmula recebe qualquer nota e transforma em probabilidades. As probabilidades somam 1 e serão grandes quando as notas forem grandes e pequenas quando as notas forem pequenas.

Figura 4 - Relação e resultados de notas inseridas na função Softmax

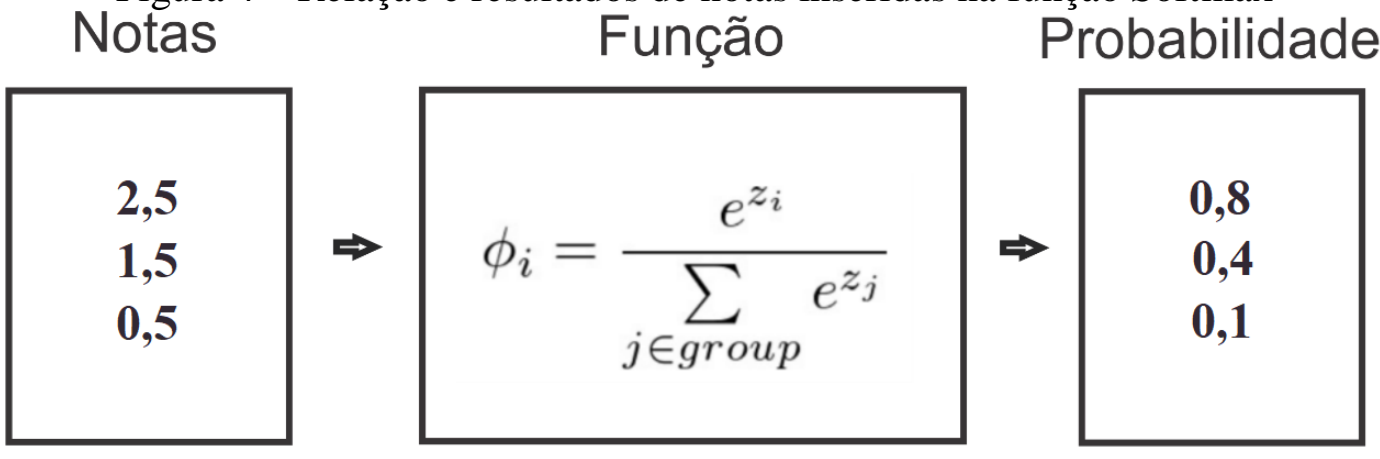

\section{Citações e referências bibliográficas}

AZEVEDO, Eduardo; CONCI, Aura. Computação Gráfica: Teoria e Prática. 1. Ed, Rio de Janeiro: Editora Campus, 2003, 384 páginas.

CRUVINEL, Freederico Borges; Tópicos de Álgebra Linear e Aplicações em Problemas de Economia e de Engenharia. Goiânia: 2013. Disponível em https://repositorio.bc.ufg.br/tede/bitstream/tde/2961/5/TCC\%20APLICA\%C3\%87\%C3\%95ES\%20DE \%20AL\%20PROFMAT\%202013.pdf. Acesso em 25 de julho de 2017.

CRUZ, L.F; Gonçalves, E.M; CHUEIRI, V.M.M; Introdução ao Estudo da Álgebra linear. São Paulo: 2012. Disponível em http://culturaacademica.com.br/_img/arquivos/Introd\%20ao\%20Est\%20\%20Algebra\%20Linear\%20p\% 20Livraria\%20Virtual\%20(2).pdf. Acesso em 19 de dezembro de 2017.

HEFEZ, Abramo; FERNANDEZ, Cecília S. Introdução à Álgebra Linear. 2. ed. Rio de Janeiro: Editora SBM, 2016, 271 páginas.

Google, Udacity. Deep Learning. 2011 - 2017. Disponível em https://br.udacity.com/course/deeplearning--ud730/. Acesso em 07 de julho de 2017.

PAAR, Christof; PELZI, Jan. Understanding Cryptography: A Textbook for Students and Practitioners. Edição de 2010, Berlim: Editora, 2014, 372 páginas.

TAING, Seamleng; Application of Linear Algebra in Electrical Circuits. 2011. Disponível em https://sites.math.washington.edu/ king/coursedir/m308a01/Projects/m308a01-pdf/taing.pdf. Acesso em 04 de julho de 2017. 
Title - Numerical linear algebra: applications in computational methods and their importance to engineering

\begin{abstract}
The present work aims to validate the importance of linear algebra in computational methods and engineering courses, showing not only theoretical aspects but also practical applications of the discipline. Although the concepts of this area of mathematics are widely diffused in their theoretical field, their applicability often goes unnoticed, especially in the initial semesters of engineering courses, thus contributing to a difficult visualization of their usability. In this way, the need arises to demonstrate how the concepts apply in solving problems faced by engineers. In fact, these applications can be seen not only in computing, but also in other engineering. Therefore, it is essential to approach some of these uses, as well as their respective methods of implementation.
\end{abstract}

Keywords - Algebra; Engineering; Computing; Application.

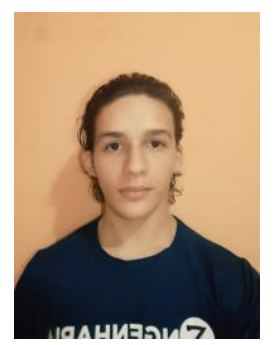

José Rhuan de Souza Pereira, técnico em informática e discente do $4^{\circ}$ semestre (2017.2) do curso de Engenharia da Computação na Universidade Federal do Ceará. Principais áreas de interesse: inteligência artificial, tecnologias web e segurança da informação, além de entusiasta por hardware e internet das coisas.

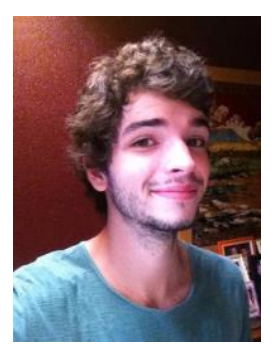

Renato Brito Costa, discente do $3^{\circ}$ semestre (2017.2) do curso de Engenharia de Computação na Universidade Federal do Ceará. Áreas de interesse: sistemas embarcados, tecnologias web, mobile e internet das coisas. 